\title{
Discrete Spectrum of Bifurcation of Exact Solutions for Stationary Longitudinal Waves in the Flow of Perfect Fluid Around a Circular Body of a Large Radius
}

\author{
R. N. Ibragimov * \\ ${ }^{1}$ GE Global Research 1 Research Circle Niskayuna, NY 12309, USA
}

\begin{abstract}
We consider a free boundary problem for incompressible perfect fluid circulating around a circle $\Gamma$ of a large radius, i.e. within a central gravity field. The outward curve $\gamma$ is a free boundary to be sought. We assume that the flow, which is confined between $\Gamma$ and $\gamma$, is irrotational. The centrifugal force caused by the circulation of the flow makes the fluid go outward. We show that there exist stationary waves, which are periodic oscillations of the fluid and are exact solutions corresponding to bifurcating branches emanating from trivial solution. In the frame of the problem in question, the wave number can take only the integer multiplies of $2 \pi$. This makes the spectrum discrete, which makes a crucial difference with the problem for flows in an infinite domain over the straight line or plane (i.e. the ocean problem). To prove the existence of exact solutions, the method of conformal mapping is used. By this device the free boundary problem is transformed into a boundary value problem in a fixed domain.
\end{abstract}

Keywords and phrases: free boudary, bifurcation phenomena, spectral values

Mathematics Subject Classification: 35R35, 35A01, 76B03

\section{Introduction}

Planetary waves are large-scale perturbations of the atmospheric dynamic structure that extend coherently around a full longitude circle. They are important because they have significant influence on the wind speeds, temperature, distribution of ozone, and other characteristics of the middle atmosphere structure. They also play an important role in global climate control and weather prediction, see [27]. In oceanographic applications, understanding of the atmospheric processes mechanisms have greatly increased due to microstructures measurements over the past two decades. In terms of mathematical modeling, the large-scale atmospheric dynamics is usually described by moving air masses on a sphere or circle by means of three and two dimensional Navier-Stokes or Euler equations a thin rotating spherical shell (see e.g. [7], [8]; [23], [24]; [12], [13], [16], [18], [16]; [6]) or within the theory of shallow water approximation ([21]; [28]; [3]; [19]; [15]). In particular, a large-scale two-dimensional modeling with the inclusion of a spherical shape can be associated e.g. with the eastward moving, wave of warm water, known as a Kelvin wave that can be seen traveling eastward along the equator as shown in the left Panel

${ }^{*}$ Corresponding author. E-mail: ibrranis@gmail.com 
of Figure 1 ( see [9]). Another spectacular example of circulating waves is demonstrated on the right panel of Figure 1 showing a jet stream that follows a hexagon-shaped path at the north pole of Saturn. The hexagon was hidden in darkness during the winter of Saturn's long year, a year that is equal to about 29 Earth years. But as the planet approached its August 2009 equinox and signaled the start of northern spring, the hexagon was revealed to Cassini's cameras. The images were obtained at a distance of approximately 764,000 kilometers (475,000 miles) from Saturn. The smallest resolved features at the latitude of the hexagon have a horizontal scale of approximately 100 kilometers. Recent laboratory experiments in Aguiar et al. [1] suggest that the observed Saturn's North Polar Hexagon might result from the stabilization of a standing waves caused by the difference in angular velocity. However, because of the complex atmospheric structure in Saturn, the provided experiments do not provide the clear answers and these waves and the six-sided shape of the jet stream remain a mystery up to the date [2].

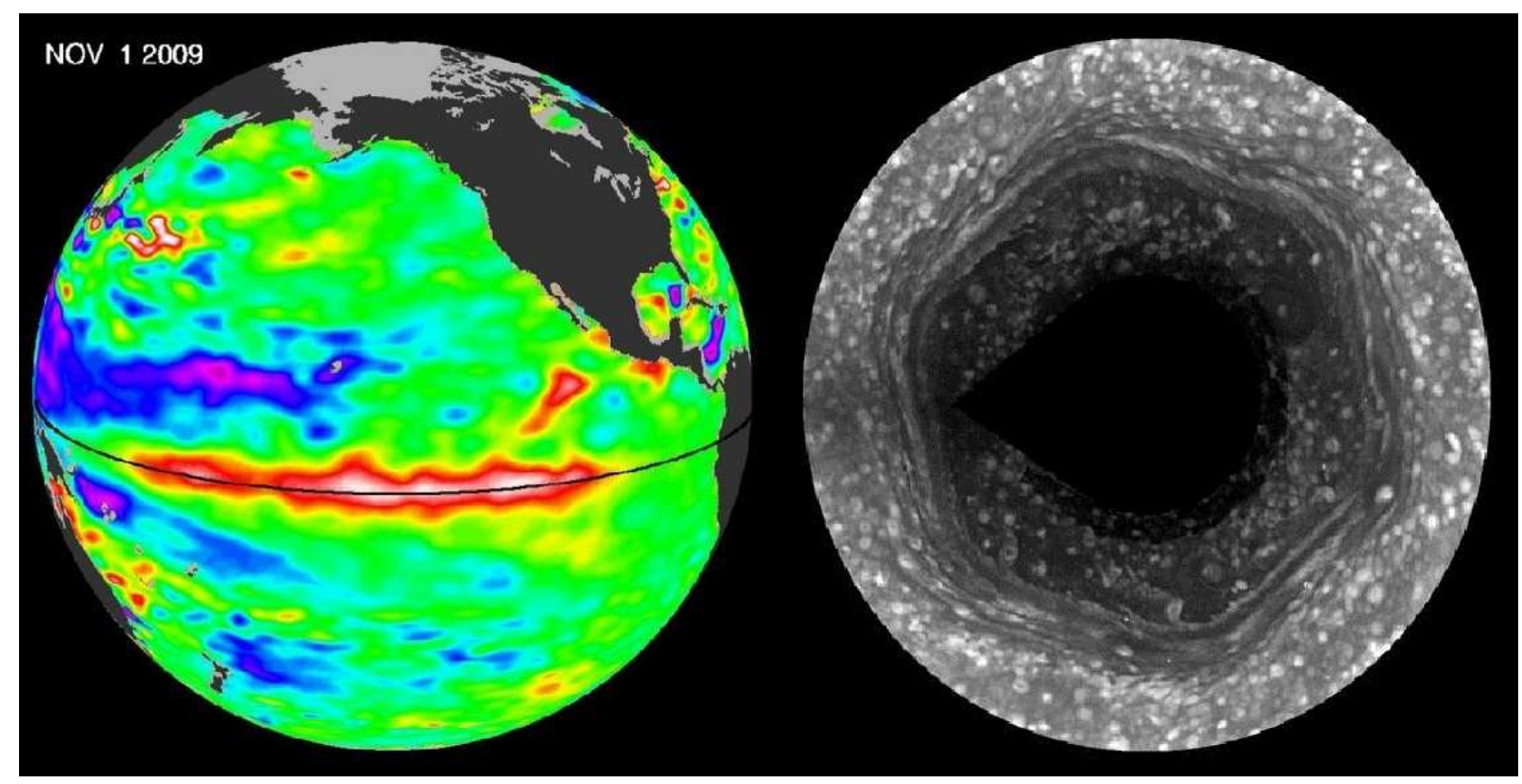

Figure 1. Left: Sea-level height data from November 2009 showing the dynamics of warm water known as Kelvin waves that can be seen traveling eastward along the equator (black line) in Nov. 01, 2009 image. El Ninos form when trade winds in the equatorial western Pacific relax over a period of months, sending Kelvin waves eastward across the Pacific like a conveyor belt. Image credit: NASA/JPL. Right: Image from Cassini, made possible only as Saturn's north pole emerged from winter darkness, shows new details of a jet stream that follows a hexagon-shaped path and has long puzzled scientists (see $[5])$.

The primary focus in this paper is to a show the existence of stationary wave solutions associated with the stationary flow of perfect fluid with a free boundary $\gamma$ circulating around a solid circle $\Gamma$ (see Fig. 2 ). We assume that the radius of the circle $\Gamma$ is sufficiently large so that the gravity is directed to the center of $\Gamma$ and so the the problem to be considered here can be associated with a longitudinal large-scale atmospheric wave motion within a central gravity field (see also [13], [17]; [26]). In particular, as has been discussed in [14], [15] and [17], the mathematical model can be derived from the assumption that the atmosphere is approximated by a perfect fluid and its motion is irrotational and pressure on a free boundary is constant. It is also postulated that the fluid depth is small compared to the radius of the circle $\Gamma$ and the gravity vector is directed to the center of the circle. 
It has been shown in [19] that, in the first approximation, shallow water equations represent the mathematical theory that can be used to investigate the atmospheric waves in the form of progressive $2 \pi$-periodic solitary waves (which is similar to the case of a flat bottom considered in the modeling of the fluid flows in channels in [10]). However, as has been discussed in [19], this theory does not reveal the role the role of an undisturbed level of the fluid surface which is needed to determine the precision of the first approximation. A higher order approximation was investigated in [19] and it was shown that the corresponding theory of a higher-order shallow approximation admits two functionally independent systems, while the classical problem for the flat bottom admits only one system. This splitting phenomena was a key factor in focusing on the question on existence of exact solution for a such planetary-scale model, which is investigated in the frame of the present work.

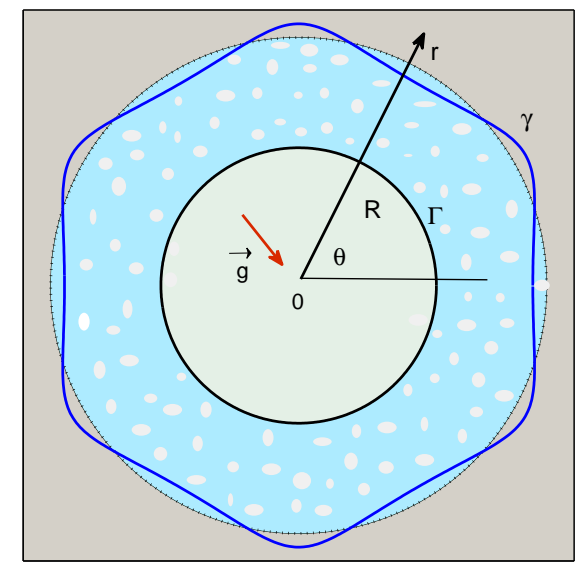

FiguRE 2. Schematic showing a longitudinal atmospheric motion circulating around the Earth.

We will show that there exist exact solutions to the problem in the form of $2 \pi$-periodic stationary oscillations of a fluid circulating around a solid circle $\Gamma$. To this end, we will first perform the transformation from the physical flow domain to the plane of the complex potential. Further, we will formulate the problem in terms of operator equations and apply the bifurcation theory to the resulting operator equations. Finally, we shall prove the existence of exact solutions in a neighborhood of a trivial solution.

\section{The model}

We consider a stationary flow of perfect fluid with a free boundary around a circle within a central gravity field so that the model can be associated with a longitudinal atmospheric motion around a celestial body, e.g. the Earth. We consider a plane through an equator of a celestial body and a two-dimensional flow in this plane. We assume that the flow is enclosed by two closed Jordan curves $\Gamma$ and $\gamma$, as shown schematically in Figure 2. $\Gamma$ is an equator of a celestial body and $\gamma$ is a free boundary, which is outside $\Gamma$.

We use the following notation: $R$ is a radius of the circle $\Gamma, \theta$ is a polar angle, $r$ is a distance from the origin, $h$ is the undisturbed level of the fluid above $\Gamma$ and $\eta(\theta)$ is the level of disturbance of the free boundary. The fluid lies in a doubly connected domain enclosed by $\Gamma$ and $\gamma$, i.e. in the domain

$$
\Omega_{(r, \theta)}=\{(r, \theta): 0 \leqslant \theta \leqslant 2 \pi, \quad R \leqslant r \leqslant R+h+\eta(\theta)\} .
$$

The velocity field $u=\left(u_{r}, u_{\theta}\right)$ in polar coordinates satisfies Euler's equations and the condition $u_{r}=0$ on $\Gamma$. The kinematic condition on the free boundary $\gamma$ is assumed. Namely, the velocity is tangent to the 
free boundary. The force of gravity $g$ is assumed to be constant and directed to the center of the circle $\Gamma$. For the sake of simplicity, a fluid is assumed to be perfect and its motion is supposed to be irrotational. The effects of the surface tension is neglected. We suppose also that every wave has an axis of symmetry and the pressure on the free boundary is a constant.

We introduce the stream function $\psi$ as follows:

$$
u_{r}=-\frac{\psi_{\theta}}{r}, \quad u_{\theta}=\psi_{r},
$$

where the subscripts of $\psi$ denote the partial derivatives.

We next introduce the following nondimensional variables:

$$
\theta=\widehat{\theta}, \quad r=R+h \widehat{r}, \quad u=U \widehat{u}, \quad \psi=U h \widehat{\psi}, \quad \varepsilon=\frac{h}{R}, \quad \alpha=(1+\varepsilon r) e^{i \theta} .
$$

In this notation, the problem is to find a closed Jordan curve $\gamma(\theta)$ and a stream function $\psi(r, \theta)$ satisfying the following conditions:

$$
\begin{gathered}
\varepsilon^{2} \psi_{\theta \theta}+(1+\varepsilon r)^{2} \psi_{r r}+(1+\varepsilon r) \psi_{r}=0 \quad(0<r<1+\eta), \\
\psi(\theta, 0)=0 \\
\psi(\theta, 1+\eta(\theta))=1 \\
\varepsilon^{2}(1+\varepsilon r)^{-2} \psi_{\theta}^{2}+\psi_{r}^{2}+2 F^{-2} \eta=2 b, \quad(r=1+\eta),
\end{gathered}
$$

where $b=U^{2} \widehat{b}$ is the Bernoulli constant and

$$
F=\frac{U}{\sqrt{g h}}
$$

is the Froude number. It is supposed in what follows that $\theta \in[0,2 \pi]$ and $r \in[R, h(t, \theta)]$. The restriction $\theta \in[0,2 \pi]$ appears for the following reason: the velocity potential $\varphi$ can be introduced by the analyticity of the complex potential

$$
\omega(\alpha)=\varphi+i \psi,
$$

where $\alpha$ introduced by (2.3) is the independent complex variable. Correspondingly, the complex velocity $d \omega / d \alpha$ is a single-valued analytic function of $\alpha$, although $\omega$ is not single-valued. In fact, when we turn around the bottom $r=R$ once, $\varphi$ increases by $-\int_{0}^{2 \pi} \frac{\partial \psi}{\partial r}(R, \theta) d \theta$, which has a positive sign by the maximum principle (Hopf's lemma). Hence, if we remove the width of annulus region $\theta=0, r \in\left[R, R+h_{0}\right]$, then at every point $(r, \theta)$, the complex potential $\omega(\alpha)$ is a single-valued analytic function.

In terms of the complex potential $\omega$, we can easily find a simple solution of the model $(2.4)-(2.7)$ given by

$$
\eta=0, \quad \psi \equiv \omega_{0}(\alpha)=\frac{\operatorname{Re}(\log \alpha)}{\log (1+\varepsilon)} .
$$

This solution represents a fluid motion with undisturbed free surface and can be called a trivial solution.

We will prove that there exist progressive wave solution in a neighborhood of the trivial solution (2.10). We remark that we show the existence of progressive waves by the bifurcation theory. In utilizing the theory, we take the propagation speed as a bifurcation parameter. This fact provides for distinction between our problem and the problem for flows in an infinite domain over the straight line or plane (i.e. the ocean problem). Indeed, the wave number ranges over all real numbers in the case of the ocean 
problem. On the contrary, in our problem, the wave number can take only the integer multiplies of $2 \pi$. This makes the spectrum discrete, whence we can use the bifurcation theory (see [11]).

Thus, our model (2.4) - (2.7) is a bifurcation problem for two unknowns are $\psi, \eta$ and three parameters $\varepsilon, F$ and $b$. We aim to show that there are exist nontrivial solutions of the free boundary problem (2.4) - (2.7) bifurcating from $\omega_{0}(\alpha)$.

\section{Plane of the complex potential}

We first rewrite the model $(2.4)$ - $(2.7)$ in the $(x, y)$ - plane by introducing the transformation

$$
\alpha=e^{-i \widetilde{\alpha}},
$$

where $\widetilde{\alpha}=x+i \epsilon y$ and $\epsilon=\log (1+\varepsilon)$. By defying the free boundary disturbance in $(x, y)-$ plane as $\widetilde{\eta}$, one can see that the previous and new variables are related by the following formulae:

$$
1+\varepsilon r=e^{\epsilon y}, \quad \theta=-x, \quad \eta=\frac{e^{\epsilon}\left(e^{\epsilon \widetilde{\eta}}-1\right)}{e^{\epsilon}-1} .
$$

Thus the transformation (3.1) maps the original domain onto $\Omega_{(x, y)}$ given by

$$
\Omega_{(x, y)}=\{(x, y): x \in(-2 L, 0), \quad y \in(0,1+\widetilde{\eta}(x))\},
$$

where $-2 L$ is a wave period in the domain $\Omega_{(x, y)}$. Without loss of generality we assume that $\widetilde{\eta}$ is symmetric about the line $x=-L$, i.e.,

$$
\int_{-L}^{0} \tilde{\eta} d x=0 .
$$

Since

$$
\frac{d \omega}{d \alpha}=\frac{d \omega}{d \widetilde{\alpha}} e^{-\epsilon y} e^{i x}
$$

the nondimensionalized model (2.4) - (2.7) can be written in the domain $\Omega_{(x, y)}$ as follows:

$$
\begin{gathered}
\epsilon^{2} \psi_{x x}+\psi_{y y}=0 \quad(0<y<1+\widetilde{\eta}(x)), \\
\psi(x, 0)=0, \quad \psi(x, 1+\widetilde{\eta}(x))=1, \\
e^{-2 \epsilon y}\left(\psi_{x}^{2}+\frac{1}{\epsilon^{2}} \psi_{y}^{2}\right)+2 \frac{e^{\epsilon}\left(e^{\epsilon \widetilde{\eta}}-1\right)}{\left(e^{\epsilon}-1\right)^{3}} F^{-2}=2 b \quad(y=1+\widetilde{\eta}(x)) .
\end{gathered}
$$

Additionally the problem (3.4) - (3.7) for two unknowns $(\psi(x, y), \widetilde{\eta}(x))$ has to be solved subject to the periodicity condition $\psi(0, y)=\psi(-2 L, y)$ and $\psi_{x}(0, y)=\psi_{x}(-2 L, y)$. Since the stream function $\psi$ is defined in an unknown domain, it is advantageous to consider $(\psi, \varphi)$ as new dependent variables of the problem. By this device, the free boundary is represented by a line $\psi=1$ while the bottom is given by $\psi=0$. Then $\varphi$ changes from 0 to $2 K$, where $K$ is defined by the periodicity condition.

We next introduce the Levi-Civita functions $t$ and $\lambda$ (see [22]) by

$$
\frac{d \omega}{d \widetilde{\alpha}}=\epsilon^{-1} e^{-i(t+i \lambda)}
$$

and differentiate the dynamic boundary condition (3.7) with respect to $\varphi$ along the free boundary $\psi=1$ to get

$$
e^{2(\lambda-\epsilon)}\left(e^{\epsilon}-1\right)^{2}\left(\lambda_{\varphi}-\epsilon \widetilde{\eta}_{\varphi}\right)+\frac{\epsilon^{3} e^{\epsilon}}{e^{\epsilon}-1} F^{-2} \widetilde{\eta}_{\varphi} e^{3 \epsilon \widetilde{\eta}}=0
$$


Hence the free boundary $\widetilde{\eta}$ can be written in terms of new variables $t$ and $\lambda$ as

$$
\widetilde{\eta}=\int_{0}^{\varphi} e^{-\lambda} \sin t d \varphi^{\prime}+\widetilde{\eta}(0,1),
$$

where $\widetilde{\eta}(0,1)$ is a constant of integration. Therefore, after some simplifications of Eq. (3.9), the model (3.4) - (3.7) can be written as the following boundary value problem:

$$
\begin{gathered}
t_{\varphi \varphi}+t_{\psi \psi}=0 \quad(0<\psi<1), \\
t(\varphi, 0)=1, \\
F^{(1)}=\gamma^{*}\left(t_{\psi}+\epsilon e^{-\lambda} \sin t\right)-e^{-3 \lambda} e^{3 \epsilon \int_{0}^{\varphi} e^{-\lambda} \sin t d \varphi^{\prime}} \sin t=0 \quad(\psi=1),
\end{gathered}
$$

where the parameter $\gamma^{*}$ is defined as

$$
\gamma^{*}=\frac{\left(e^{\epsilon}-1\right)^{3}}{\epsilon^{3}} e^{-3 \epsilon(1-\widetilde{\eta}(0,1))} F^{2} .
$$

Using the terminology in [25], we call $\gamma^{*}$ the Nekrasov parameter (Nekrasov, [25] was the first mathematical paper which proves the existence of solutions to the classical water wave problem over a flat bottom. It was proved independently in a later work [10] that the theorem of existence of solitary waves, in the shallow water approximation, holds true ). In order to express the periodicity condition in the new variables, we utilize the Beale's approach (see [4]), which has also been incorporated and adjusted in [20] to describe a periodic atmospheric motion in shallow water approximation. In the frame of the present modeling, following [4] and [20], we can show that

$$
\frac{1}{\epsilon} \int_{-L}^{0}(1+\widetilde{\eta}(x)) d x=\int_{0}^{K} \int_{0}^{1} e^{-2 \lambda} d \varphi d \psi=\left[\int_{0}^{K} e^{-\lambda} d \varphi\right]_{\psi=1},
$$

in which the Jacobian of transformation from the variables $(x, y)$ to $(\varphi, \psi)$ was computed to be equal to $e^{2 \lambda} / \epsilon$. The latter two equations in (3.15) represent the condition of periodicity. We next use the following renormalization:

$$
\mu=\frac{\pi}{K}, \quad \widehat{\varphi}=\mu \varphi
$$

so that $\widehat{\varphi}$ moves from 0 to $2 \pi$. Hereafter the symbol "hat" is omitted. We note that the Cauchy-Riemann equations

$$
\lambda_{\psi}=\mu t_{\varphi}, \quad \lambda_{\varphi}=-\frac{1}{\mu} t_{\psi}
$$

enable us to express the function $\lambda$ in terms of $t$ as

$$
\lambda(\varphi, \psi)=\mu \int_{1}^{\psi} t_{\varphi} d \psi^{\prime}-\frac{1}{\mu} \int_{0}^{\varphi} t_{\psi} d \varphi^{\prime}+c(0,1),
$$

where $\mu$ is defined by $(3.16)$ and $c(0,1)$ is a constant of integration, which is determined by the condition of periodicity (3.15) as follows:

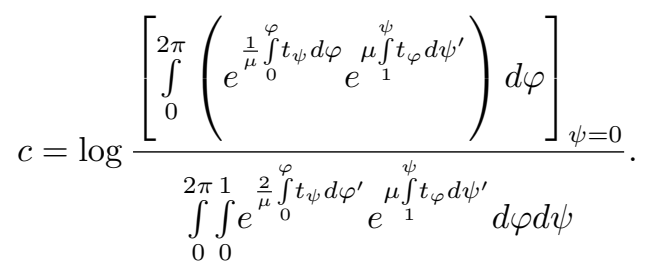


We look for odd and periodic solutions in $\varphi$ of the problem (3.11) - (3.13), i.e. in the form

$$
t=\sum_{n=1}^{\infty} t_{n}(\psi) \sin (n \varphi) .
$$

We next define two normed Banach spaces $\mathcal{B}$ and $\widetilde{\mathcal{B}}$ as follows:

$$
\begin{gathered}
\mathcal{B}=\left\{t(\varphi, \psi): \sum_{n=1}^{\infty} \sup _{0<\psi<1}\left|t_{n}(\psi)\right|<\infty,\|t\|_{\mathcal{B}}=\sum_{n=1}^{\infty}\left|t_{n}(\psi)\right|_{\infty}\right\}, \\
\widetilde{\mathcal{B}}=\left\{t(\varphi, \psi): \sum_{n=1}^{\infty} \sup _{0<\psi<1}(1+n)\left|t_{n}(\psi)\right|<\infty,\|t\|_{\widetilde{\mathcal{B}}}=\sum_{n=1}^{\infty}(1+n)\left|t_{n}(\psi)\right|_{\infty}\right\}
\end{gathered}
$$

In the next section, we formulate the main result on bifurcation of the non-trivial solution from the trivial one given by (2.10) in a some small neighborhod

\section{Bifurcation phenomena}

We introduce the following notation:

$$
\gamma_{k}=\frac{1}{k \operatorname{coth} k+\epsilon}, \quad \delta=\gamma_{k}-\gamma,
$$

where $k=1,2,3, \ldots$

Theorem 1. For any fixed value $\epsilon>0$ and any arbitrary positive integer $k$, there exists a constant a, such that for all $\delta$ with $|\delta|<a$, the system (3.11) - (3.13) subject to the periodicity condition (3.15) has one-parameter family of solutions $(t(\delta), \mu(\delta))$ near $(0,1)$. In particular, the following inequality holds:

$$
\|t\|_{\widetilde{\mathcal{B}}}+|\mu-1| \leqslant \beta(\epsilon) \delta
$$

where $\beta$ is a constant.

In order to prove the Theorem 1 , it is convenient to rewrite the system (3.11) - (3.13) in the following operator notation:

$$
\begin{gathered}
F^{(1)}\left(t_{(+)}, \mu\right)=\gamma^{*}\left(N_{\mu} t_{(+)}+\epsilon e^{c+\frac{\nu}{\mu}} \sin t_{(+)}\right)-e^{\frac{3 \epsilon}{\mu} \int_{0}^{\varphi}\left(e^{c+\frac{\nu}{\mu}} \sin t_{(+)}\right) d \varphi^{\prime}} e^{3\left(c+\frac{\nu}{\mu}\right)} \sin t_{(+)}=0, \\
F^{(2)}\left(t_{(+)}, \mu\right)=\mu-\frac{\epsilon^{2}}{\pi}\left[\int_{0}^{2 \pi}\left(e^{c+\frac{\nu}{\mu}} \sin t_{(+)}\right) d \varphi\right]_{\psi=0}=0,
\end{gathered}
$$

where we use the following notation:

$$
\nu=\int_{0}^{\varphi} N_{\mu} t_{(+)} d \varphi,
$$

in which $t_{(+)}=t(\varphi, 1)$ and

$$
\left.N_{\mu} t_{(+)} \equiv t_{\psi}\right|_{\psi=1}
$$

is the normal derivative, so that

$$
\left(N_{\mu} t_{(+)}\right)_{n}=\mu n \operatorname{coth}(\mu n) t_{(+) n}
$$

We note that it is enough to solve system (4.3) - (4.4) for $t_{(+)}$and $\mu$ only since $t(\varphi, \psi)$ is well defined by $t_{(+)}$due to equations (3.11) and (3.12). 
The possibility of the existence of the only trivial solution can be excluded by means of the following

Lemma 1. For any $t, t_{1} \in \widetilde{\mathcal{B}}$ and $0 \leqslant \mu \leqslant 1$, the following three inequalities hold:

$$
\begin{aligned}
\|\nu\|_{\mathcal{B}} & \leqslant 2\|t\|_{\widetilde{\mathcal{B}}}, \quad\|\sin t\|_{\mathcal{B}} \leqslant \sinh \|t\|_{\widetilde{\mathcal{B}}}, \\
\left\|\cos t-\cos t_{1}\right\|_{\mathcal{B}} & \leqslant \sinh \left\{\|t\|_{\widetilde{\mathcal{B}}}+\left\|t_{1}\right\|_{\widetilde{\mathcal{B}}}\right\}\left\|t-t_{1}\right\|_{\widetilde{\mathcal{B}}} .
\end{aligned}
$$

Proof. The first two inequalities follow directly from the presentation

$$
\nu=-\sum_{n=1}^{\infty} \operatorname{coth}\left(t_{n}\right)[\cos (n \varphi)-1],
$$

in which we use the result that the $n^{\text {th }}$ Fourier component of the normal derivative is calculated as in formula (4.7). The relation (4.10) implies that $n$

$$
\|\nu\|_{\mathcal{B}} \leqslant 2 \sum_{n \geqslant 1}\left|n \operatorname{coth}(n) t_{n}\right| \leqslant 2\|t\|_{\widetilde{\mathcal{B}}} .
$$

The second inequality in (4.8) is a sequence of the following estimate:

$$
\|\sin t\|_{\mathcal{B}} \leqslant \sum_{n \geqslant 1} \frac{\|t\|_{\mathcal{B}}^{2 n-1}}{(2 n-1) !}=\sinh \|t\|_{\mathcal{B}}
$$

Finally, the inequality (4.9) follows from the following estimate:

$$
\left\|\cos t-\cos t_{1}\right\|_{\mathcal{B}} \leqslant \sum_{n \geqslant 1} \frac{\left\{\|t\|_{\tilde{\mathcal{B}}}+\left\|t_{1}\right\|_{\tilde{\mathcal{B}}}^{2 n-1}\right\}\left\|t-t_{1}\right\|_{\widetilde{\mathcal{B}}}}{(2 n-1) !},
$$

which yields

$$
\left\|\cos t-\cos t_{1}\right\|_{\mathcal{B}} \leqslant \sinh \|t\|_{\mathcal{B}}+\left\|t_{1}\right\|_{\widetilde{\mathcal{B}}}\left\|t-t_{1}\right\|_{\widetilde{\mathcal{B}}}
$$

We remark that Lemma 1 guarantees that $F^{(i)}$ are a well-defined mappings from $\widetilde{\mathcal{B}}$ to $\mathcal{B}$, i.e.

$$
F^{(1)}(t, \mu): \widetilde{\mathcal{B}} \rightarrow \mathcal{B}, i=1,2
$$

and that mappings $F^{(1,2)}$ are continuous as well as their derivatives. That is to say, if $\rho$ is any element of $\widetilde{\mathcal{B}}$, it follows from Lemma 1 that

$$
\lim _{t \rightarrow t_{1}, \mu \rightarrow \mu_{1}}\left\|\left(F_{t}^{(1,2)}(t, \mu)-F_{t}^{(1,2)}\left(t_{1}, \mu_{1}\right)\right)\langle\rho\rangle\right\|_{\mathcal{B}}=0
$$

where the subscript $t$ means the Fréchet derivative.

We linearize the equation

$$
F^{(1)}(t, \mu)=0
$$

to obtain

$$
(Z \rho)_{k}=F_{t}^{(1)}(0,1)\langle\rho\rangle=[\gamma(k \operatorname{coth} k+\epsilon)-1] \rho_{k}=f_{k}
$$

where $f_{k} \in \mathcal{B}$ is the nonlinear part of Eq. (4.15) and $Z$ represents a map

$$
F_{t}^{(1)}(0,1)=Z: \widetilde{\mathcal{B}} \rightarrow \mathcal{B},
$$

where, again, the subscript $t$ means the Fréchet derivative. 


\subsection{Lyapunov method}

The proof of the Theorem 1 is based on application of the Implicit Function Theorem. It's application is valid only if the equation (4.16) has a unique solution for every $f_{k}$. However, if $\delta=0$, the operator $Z$ has one-dimensional kernel generated by functions $l_{k}(\varphi)=\sin (k \varphi)$ so that the map $Z$ does not have an inverse and the equation (4.16) does not have a unique solution. In order to remove the singularity, we utilize the Lyapunov method and use the following decomposition of the function $t$ :

$$
t=\xi \sin (k \varphi)+\sum_{n \neq k} \zeta_{n} l_{n}(\varphi),
$$

where $\xi$ is a real parameter and $\xi \sin (k \varphi) \in \operatorname{Ker}(Z)$ and $\zeta=\left(\zeta_{1}, \ldots, \zeta_{k-1}, \zeta_{k+1}, \ldots\right)$ in the equation $(4.18)$ is given by

$$
\zeta=Z^{-1} G_{k} H(\xi \sin (k \varphi)+\zeta, \mu),
$$

in which

$$
G_{k} f(\varphi)=\sum_{n \neq k} f_{n} \sin (n \varphi)
$$

and the operator $H$ is defined by

$$
H(t, \mu)=Z t-F^{(1)}(t, \mu)
$$

where $Z$ is the map defined in (4.17).

Due to Lemma 1, the equation (4.19) can be solved by the Implicit Function Theorem. Moreover, the equation (4.19) yields the following estimate for $\zeta$ :

$$
\|\zeta\|_{\widetilde{\mathcal{B}}} \leqslant c_{2}|\mu-1| \xi
$$

where $c_{2}=$ const. By the definition, the operator $H$ is written as

$$
\begin{gathered}
H(t, \mu)=\delta N_{\mu} t-\left[\gamma_{k}-\delta\right]\left\{N_{\mu} t+\gamma_{k} \epsilon t-\epsilon e^{c+\frac{\nu}{\mu}} \sin t\right\}-t+ \\
e^{\frac{3 \epsilon}{\mu} \int_{0}^{\varphi}\left(e^{c+\frac{\nu}{\mu}} \sin t\right) d \varphi^{\prime}} e^{3\left(c+\frac{\nu}{\mu}\right)} \sin t=0 .
\end{gathered}
$$

It also follows from Lemma 1 that on the free surface, the constant $c(0,1)$ has the following asymptotic:

$$
c=\frac{1}{2 \pi \mu} \int_{0}^{2 \pi} \int_{0}^{\varphi} M_{\mu} t d \varphi^{\prime} d \varphi-\frac{2}{\mu} \int_{0}^{\varphi} t d \varphi^{\prime}+\frac{1}{\pi \mu} \int_{0}^{2 \pi} \varphi t d \varphi+o\left(t^{2}\right),
$$

where

$$
\left(M_{\mu} t\right)_{n}=\frac{\mu n t_{n}}{\sinh (\mu n)} .
$$

We also note that

$$
\left\|M_{\mu} t\right\|_{\mathcal{B}} \leqslant\|t\|_{\widetilde{\mathcal{B}}}
$$

which follows from the inequality

$$
\frac{n}{\sinh n} \leqslant 1+n
$$

Because of presentations (4.23), (4.24), Lemma 1, estimates (4.22) and (4.26), the operator $H$ has the following asymptotic:

$$
\begin{gathered}
H(t, \mu)=\gamma_{k}\left(N_{\mu}-N_{1}\right) t+\delta \epsilon t- \\
-\epsilon\left(\gamma_{k}-\delta\right)\left(\frac{t \nu}{\mu}+\frac{t}{2 \pi \mu} \int_{0}^{2 \pi} \int_{0}^{\varphi} M_{\mu} t d \varphi^{\prime} d \varphi-\frac{2 t}{\mu} \int_{0}^{\varphi} t d \varphi^{\prime}+\frac{t}{\pi \mu} \int_{0}^{2 \pi} t d \varphi\right)+
\end{gathered}
$$




$$
\begin{gathered}
\frac{3 t}{2 \pi \mu} \int_{0}^{2 \pi} \int_{0}^{\varphi} M_{\mu} t d \varphi^{\prime} d \varphi-\frac{6 t}{\mu} \int_{0}^{\varphi} t d \varphi^{\prime}+\frac{3 t}{\mu}(1+\epsilon) \nu+ \\
\frac{3 t}{2 \pi \mu} \int_{0}^{2 \pi} t d \varphi+\frac{3 \epsilon t}{\mu} \int_{0}^{\varphi} t d \varphi^{\prime}+o\left(t^{2}\right) .
\end{gathered}
$$

Expressions (4.24) and (4.27) provide the following system of the bifurcation equations:

$$
\int_{-\pi}^{\pi} D_{i}(\xi, \mu) \sin (k \varphi) d \varphi=0, \quad i=1,2,
$$

where $D_{1}=H$, and $D_{2}=F^{(2)}$.

After eliminating the trivial solution $\xi=0$, the system (4.28) is reduced to the following two equations:

$$
\begin{aligned}
& 0=F^{(1)}(\xi, \mu)=\delta-P^{(1)}(\xi, \mu), \\
& 0=F^{(2)}(\xi, \mu)=\mu-P^{(2)}(\xi, \mu),
\end{aligned}
$$

where the operator $P^{(1)}$ is

$$
\begin{gathered}
P^{(1)}(\xi, \mu)=\frac{1}{\pi(k \operatorname{coth} k+\epsilon)}\left\{\frac { 3 - \epsilon ( \gamma _ { k } - \delta ) } { \mu } \left[\xi \operatorname{coth} k\left(1+\frac{1}{4 k}\right)+\right.\right. \\
\left.k \int_{-\pi}^{\pi} \zeta \cos (k \varphi) \sin (k \varphi) d \varphi\right]+\frac{1}{2 \pi \mu}\left(\frac{2 \pi \xi}{\sinh k}+\pi \int_{0}^{\varphi} M_{\mu} \zeta d \varphi^{\prime}\right)+ \\
\frac{1}{\pi \mu}\left(\frac{4 \pi^{2} \xi}{k}+\frac{2 \pi}{k} \int_{-\pi}^{\pi} \zeta \sin ^{2}(k \varphi) d \varphi+\frac{2 \pi}{k} \int_{-\pi}^{\pi} \zeta \sin (k \varphi) d \varphi\right)+ \\
\left.\frac{3 \epsilon-6+2 \epsilon\left[\gamma_{k}-\delta\right]}{\mu}\left(\frac{\pi \xi}{k}-\frac{1}{k} \int_{-\pi}^{\pi} \zeta \cos (k \varphi) \sin (k \varphi) d \varphi-\pi \int_{0}^{\varphi} \zeta d \varphi^{\prime}\right)\right\}
\end{gathered}
$$

and the operator $P^{(2)}$ is written as follows:

$$
\begin{gathered}
P^{(2)}(\xi, \mu)=\frac{\epsilon^{2}}{\pi}\left\{2 \pi+\frac{1}{\mu} \int_{0}^{2 \pi} \int_{0}^{\varphi} M_{\mu} \zeta d \varphi^{\prime} d \varphi+\right. \\
\frac{1}{2 \pi \mu} \int_{0}^{2 \pi} \int_{0}^{2 \pi} \int_{0}^{\varphi} M_{\mu} \zeta d \varphi^{\prime} d \varphi d \varphi-\frac{2}{\mu} \int_{0}^{2 \pi} \int_{0}^{\varphi} \zeta d \varphi^{\prime} d+\frac{1}{\pi \mu} \int_{0}^{2 \pi} \int_{0}^{2 \pi} \zeta d \varphi d \varphi+ \\
\frac{4 \pi \xi \operatorname{coth} k}{\mu^{2}}\left(\frac{\pi}{\sinh k}+2 \pi \xi+\xi\right)-\frac{\xi \operatorname{coth} k}{\mu^{2}} \int_{0}^{2 \pi} \int_{0}^{2 \pi} \int_{0}^{\varphi} M_{\mu} \zeta d \varphi^{\prime} d \varphi d \varphi- \\
\frac{2 \pi \xi}{\mu^{2}}\left(\frac{1}{\sinh k}+2+\frac{2}{k}\right) \int_{0}^{2 \pi} \int_{0}^{\varphi} N_{\mu} \zeta d \varphi^{\prime} d \varphi+
\end{gathered}
$$




$$
\left.\frac{2}{\mu^{2}}\left(2 \pi^{2} \xi \operatorname{coth} k-\frac{1}{k}\right) \int_{0}^{2 \pi} \int_{0}^{\varphi} \zeta d \varphi^{\prime} d \varphi\right\}+o\left(t^{2}\right) .
$$

We next define

$$
I(\xi, \mu ; \delta)=\left|\begin{array}{ll}
1-P_{\delta}^{(1)} & -P_{\mu}^{(1)} \\
-P_{\delta}^{(2)} & -1-P_{\mu}^{(2)}
\end{array}\right| .
$$

Then system (4.29) - (4.29) is solvable if $I(0,1 ; 0) \neq 0$.

Lemma 2. If $|\delta|+|\mu-1|$ is small enough, then $I(0,1 ; 0)=1$.

Proof. It follows from presentation (4.19) for $\zeta$ that the following inequality holds:

$$
\left\|\zeta_{\delta, \mu}\right\|_{\widetilde{\mathcal{B}}} \leqslant \frac{c_{3}\left\|H_{\delta, \mu}\right\|_{\mathcal{B}}}{1-c_{3}\left\|H_{\zeta}\right\|_{\mathcal{B}}}
$$

where $c_{3}=$ const. and $H_{\delta, \mu}$ is the derivative of $H$ with respect to $\delta$ and $\mu$, which is necessary to calculate the partial derivatives of operators $P^{(1)}$ and $P^{(2)}$ in formula (4.33). If $|\xi|$ as small enough, then due to Lemma 1, the presentation (4.23) for $H$ and the estimate (4.26) for $M_{\mu} t$ yield

$$
\left\|H_{\delta, \mu}\right\|_{\mathcal{B}} \leqslant c_{4}|\xi|
$$

where $c_{4}=$ const. After evaluating the partial derivatives of the operators $P^{(1)}$ and $P^{(2)}$ with respect to $\delta$ and $\mu$, one can use the latter inequalities (4.34) and (4.35) to show that all $\zeta_{k}=0$ in a sphere with a sufficiently small radius. This completes the proof.

Now, since we have shown that Eq. (4.16) has a unique solution, due to Lemma 2, the Implicit Function Theorem can be applied to the system (4.29) - (4.30) which completes the proof of the Theorem 1. That is to say, points $\gamma_{k}(k=1,2,3, \ldots)$ are the bifurcation points and the bifurcation of non-trivial solution $\delta=P^{(1)}(\xi, \mu)$ from the trivial solution $\xi=0$ takes place in some small neighborhood.

\section{Concluding remarks}

We have investigated the question of existence of exact solutions associated with a free boundary problem for incompressible perfect fluid circulating around a circle of a large radius, i.e. within a central gravity field. We have shown the existence of stationary surface waves, which correspond to bifurcating branches emanating from trivial solution. We remark that if the circulation of the flow is zero, there is no stationary solution other than one in which the free boundary is a circle. This fact is worthy of notice because even if the circulation is zero there are progressive wave solutions which are not circles. In order to prove the existence of exact solution, the method of conformal mapping has been used. By this device the free boundary problem was transformed into a boundary value problem in a fixed domain.

Acknowledgements. The author wish to express his hearty thanks to Dr. A. Barnes and Dr. P. Spaeth who kindly read the original version of the manuscript and gave me much useful comment and encouragement.

\section{References}

[1] A. Aguiar, P. Read, R. Wordsworth, T. Salter, Y, Yamazaki. A laboratory model of Saturn's North Polar Hexagon. Icarus, 206 (2), (2010), 755-763.

[2] R. Anderson, S. Ali, L. Brandtmiller, S. Nielsen, M. Fleisher., M.Q. Wind-driven upwelling in the Southern Ocean and the deglacial rise in atmospheric $\mathrm{CO}_{2}$. Science 323, (2009), 1443-1449.

[3] G. Bachelor. An Introduction to Fluid Dynamics. Cambridge University Press, Cambridge, 1967.

[4] J. Beal. Exact solitary water waves with capillary ripples at infinity. Comm. Pure. Appl. Math. 44 (2), (1991), $211-257$. 
[5] J. Bell. Hubble captures best view of mars ever obtained From Earth. HubbleSite. NASA. http://hubblesite.org/newscenter/archive/releases/2001/24, (2001), Retrieved 2010-02-27.

[6] G. Ben-Yu. Spectral method for vorticity equations on spherical surface. Math. Comput. 64, (1995), 1067.

[7] E. Blinova. A hydrodynamical theory of pressure and temperature waves and of centers of atmospheric action. C. R. Dokl. Acad. Sci.URSS 39, 257, 1943.

[8] E. Blinova. A method of solution of the nonlinear problem of atmospheric motions on a planetary scale. Dokl. Akad. Nauk SSSR N.S.110, 975, 1956 .

[9] M. Boehm, S. Lee. The implications of tropical Rossby waves for tropical tropopause cirrus formation and for the equatorial upwelling of the Brewer-Dobson circulation. J. Atmos. Sci., 60, (2003), 247-261.

[10] K. Friedrichs, D. Hyers. The existence of solitary waves. Comm. Pure. Appl. Math. 7, 1954.

[11] R. Gardner. Existence and stability of travelling wave solutions of competition models: A degree theoretic approach. J. Differential Equations, 44, (1982), 343-364.

[12] N. Ibragimov, R. Ibragimov. Integration by quadratures of the nonlinear Euler quations modeling atmospheric flows in a thin rotating spherical shell. Phys. Lett. A., 375, (2011), 3858-3865.

[13] R. Ibragimov. Nonlinear viscous fluid patterns in a thin rotating spherical domain and applications. Phys. Fluids, 23, (2011), 123102.

[14] R. Ibragimov, G. Jefferson, J. Carminati. Invariant and approximately invariant solutions of non-linear internal gravity waves forming a column of stratified fluid affected by the Earth's rotation. Int. J. Non-Linear Mech., 51, (2013), 28-44.

[15] R. Ibragimov, G. Jefferson, J. Carminati. Explicit invariant solutions associated with nonlinear atmospheric fllows in a thin rotating spherical shell with and without west-to-east jets perturbations. Springer: Anal. Math. Phys., 3 (3), (2013), 201-294.

[16] R. Ibragimov, D. Pelinovsky. Incompressible viscous fluid flows in a thin spherical shell. J. Math. Fluid Mech. 11, (2009), 60.

[17] R. Ibragimov, H. Villasenor. Energy Balance Associated With a Mixing Process at the Interface of a Two-Layer Longitudinal Atmospheric Model. J. Fluids Eng. 136(7),doi:10.1115/1.4026857, 2014.

[18] R. Ibragimov, N. Ibragimov, L. Galiakberova.R. Symmetries and conservation laws of a spectral nonlinear model for atmospheric baroclinic jets. Math. Model. Nat. Phenom.9 (5), ( 2014), 32-39.

[19] R. Ibragimov, L. Guang. Splitting phenomenon of a higher-order shallow water theory associated with a longitudinal planetary waves. Dyn. Atmos. Oceans Volume 69, (2015), 1-11.

[20] R. Ibragimov. On the tidal motion around the Earth complicated by the circular geometry of the ocean's shape without Coriolis forces. Math. Phys. Anal. Geom. 4, (2001), 51-53.

[21] D. Iftimie, G. Raugel, G. Some results on the NS equations in thin three-dimensional domains. J. Differ. Equations 169, 281, 2001.

[22] T. Levi-Civita, T. Determination rigoureuse des ondes permanent d'ampleur finie. Math. Ann. 93, (1925), $256-314$.

[23] L. Lions, R. Teman, S. Wang. S. On the equations of the large-scale ocea., Nonlinearity 5, 1007, 1992 .

[24] L. Lions, R. Teman, S. Wang. New formulations of the primitive equations of atmosphere and applications. Nonlinearity $5,237,1992$.

[25] Nekrasov, A.I., 1952: Exact theory of the uniform waves on the surface of hard liquid, USSR Academy of Science, Moscow.

[26] H. Okamoto. Nonstationary free boundary problem for perfect fluid with surface tension. J. Math. Soc. Japan 38 (3), 1986.

[27] C. Summerhayes, S Thorpe. Oceanography: An Illustrative Guide. Wiley, New York, 1996.

[28] W. Weijer, F. Vivier, S. Gille, H. Dijkstra, H. Multiple oscillatory modes of the Argentine Basin. Part II: The spectral origin of basin modes. J. Phys. Oceanogr. 37, 2869, 2007. 